\title{
Results on mass composition of cosmic rays as measured with LOFAR
}

A. Corstanje, ${ }^{a, b, *}$ S. Buitink, ${ }^{a, b}$ H. Falcke, ${ }^{b, c, d}$ B.M. Hare, ${ }^{e}$ J.R. Hörandel, ${ }^{b, d, a}$

T. Huege, ${ }^{f, a}$ G. K. Krampah, ${ }^{a}$ P. Mitra, ${ }^{a}$ K. Mulrey, ${ }^{a}$ A. Nelles, ${ }^{g, h}$ H. Pandya, ${ }^{a}$ J. P. Rachen, ${ }^{a}$ O. Scholten, ${ }^{i}$ S. ter Veen, ${ }^{c}$ S. Thoudam, ${ }^{j}$ G. Trinh $^{k}$ and T. Winchen ${ }^{l}$

${ }^{a}$ Vrije Universiteit Brussel, Astrophysical Institute, Pleinlaan 2, 1050 Brussels, Belgium

${ }^{b}$ Department of Astrophysics/IMAPP, Radboud University Nijmegen

P.O. Box 9010, 6500 GL Nijmegen, The Netherlands

${ }^{c}$ Netherlands Institute for Radio Astronomy (ASTRON)

Postbus 2, 7990 AA Dwingeloo, The Netherlands

${ }^{d}$ Nikhef, Science Park Amsterdam, 1098 XG Amsterdam, The Netherlands

${ }^{e}$ University of Groningen, Kapteyn Astronomical Institute, Groningen, 9747 AD, Netherlands

${ }^{f}$ Institut für Astroteilchenphysik, Karlsruhe Institute of Technology (KIT)

P.O. Box 3640, 76021, Karlsruhe, Germany

${ }^{g}$ DESY, Platanenallee 6, 15738 Zeuthen, Germany

${ }^{h}$ ECAP, Friedrich-Alexander-University Erlangen-Nürnberg, 91058 Erlangen, Germany

${ }^{i}$ Interuniversity Institute for High-Energy, Vrije Universiteit Brussel

Pleinlaan 2, 1050 Brussels, Belgium

${ }^{j}$ Department of Physics, Khalifa University, P.O. Box 127788, Abu Dhabi, United Arab Emirates

${ }^{k}$ Department of Physics, School of Education, Can Tho University Campus II

3/2 Street, Ninh Kieu District, Can Tho City, Vietnam

${ }^{l}$ Max-Planck-Institut für Radioastronomie, Auf dem Hügel 69, 53121 Bonn, Germany

E-mail: a.corstanje@astro.ru.nl

We present an updated analysis of the mass composition of cosmic rays in the energy range of $10^{16.8}$ to $10^{18.3} \mathrm{eV}$. It is based on measurements with the LOFAR telescope of the depth of shower maximum, $X_{\max }$. We review the improvements to the simulation-based reconstruction setup, as well as the selection method to obtain a minimally biased $X_{\max }$ dataset. Results include estimates of the mean and standard deviation of the $X_{\max }$ distribution. A statistical analysis at distribution level has been done as well, using a four-component model of light to heavy nuclei. It confirms our previous results showing a significant low-mass fraction in this energy range.

The radio technique has advanced enough that multiple observatories are publishing results on $X_{\max }$. As the array layouts and methods vary, it is interesting to compare the approaches, in light of the observed differences in the $X_{\max }$ results. We therefore show additional information on bias tests used in the $X_{\max }$ reconstruction and sample selection process.

$37^{\text {th }}$ International Cosmic Ray Conference (ICRC 2021)

July 12th - 23rd, 2021

Online - Berlin, Germany

\footnotetext{
${ }^{*}$ Presenter
} 


\section{Introduction}

The LOFAR radio telescope [1] has been measuring radio signals from cosmic-ray air showers since 2011, in the frequency range 30 to $80 \mathrm{MHz}$. Featuring nearly 300 usable antennas in a $320 \mathrm{~m}$ diameter, it is at present still the most dense radio array used for cosmic-ray research. Measurements of the radio pulse energy footprint at this high resolution allow for estimating $X_{\max }$ per air shower to about $20 \mathrm{~g} / \mathrm{cm}^{2}$ [2], which is well suitable for mass composition analysis, as already demonstrated in 2016 [3].

Recently, we have published an updated mass composition analysis [4]. Various improvements to the previous analysis have lowered the systematic uncertainties on $X_{\max }$ and on primary energy. For $X_{\max }$, the most important improvements are the use of local atmospheric data for each air shower [5], and a better detector description for the fiducial sample selection (bias tests, see Sect. 3). For the primary energy, the main innovation has been the absolute calibration of the antennas and signal chain [6], allowing to use the radio signals directly for the energy estimate [7], independent of the particle detectors.

We report the main results on mean and standard deviation of $X_{\max }$, and an element-based mass composition analysis, together with additional material on tests for bias in $X_{\max }$. Further details can be found in [4].

\section{Reconstruction of the shower maximum $X_{\max }$}

We reconstruct $X_{\max }$ for each measured shower using an ensemble of around 30 simulations done with Corsika and CoREAS (v7.7100) [8,9]. The radio footprint of each simulation is fitted to the data, with an overall scale factor and a core position as free parameters. This gives a minimum in fit $\chi^{2}$ as a function of $X_{\max }$, which is taken as our $X_{\max }$ estimate. To ensure the simulated ensemble spans the natural $X_{\max }$ range, we take both proton and iron primaries, and use CONEX (v4.3700) [10] to pre-select showers for their $X_{\max }$, from a set of 450 showers with a proton, and 150 with an iron primary.
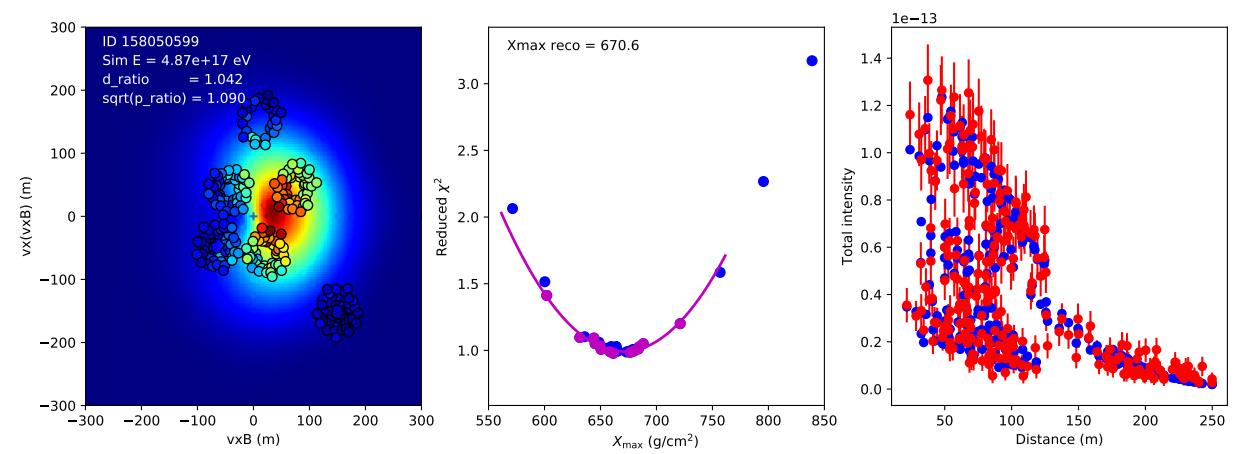

Figure 1: Left: Pulse energy in an example of a measured shower (circles), and in the best-fitting simulated shower (background colors). Middle: fit quality versus $X_{\max }$, where a parabolic fit determines the $X_{\max }$ estimate for this shower; points shown in magenta form a 'lower envelope' and contribute to this fit. Right: a radial distribution of measured and simulated pulse energy. 

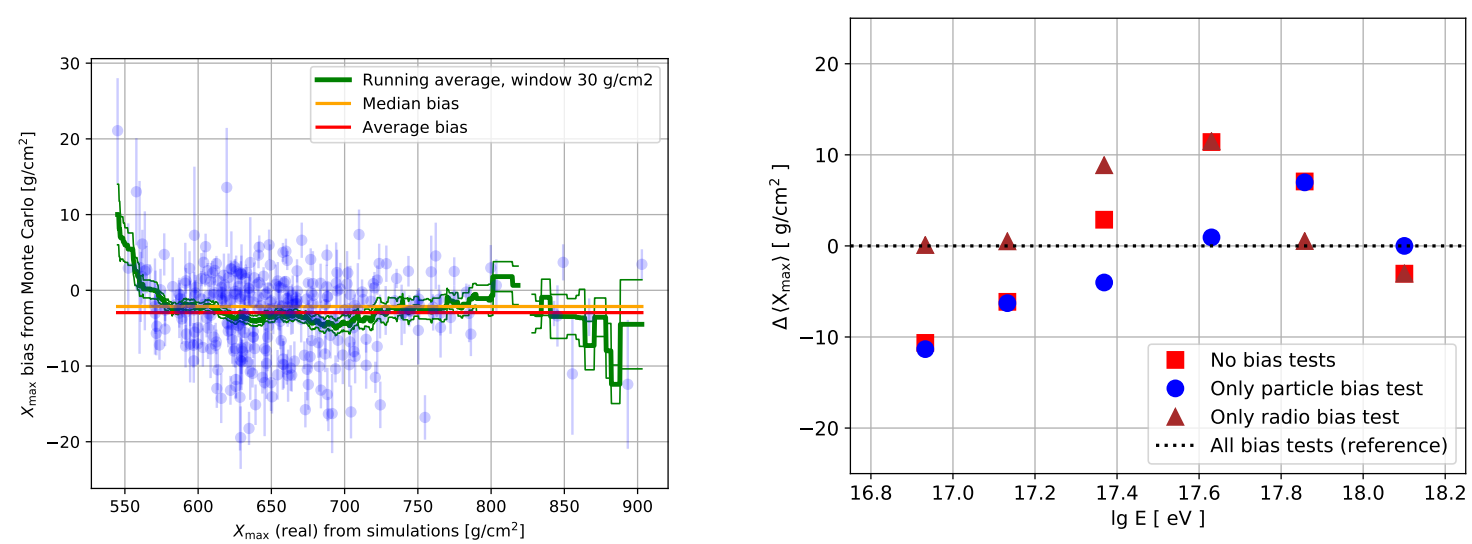

Figure 2: Left: Test for bias on reconstructed $X_{\max }$ per shower. A running average is shown (green), as well as an overall average (red) and median (yellow). Right: Left: the difference in the average $X_{\max }$ versus energy (see Sect. 4), when applying selection criteria based on only the particle bias test, only the radio bias test, or neither.

An example of this fit reconstruction is shown in Fig. 1. It shows the best-fitting shower (left panel) as well as the $X_{\max }$ estimate from the set of reduced $\chi^{2}$ values from the ensemble. A parabola is fitted to a region in $X_{\max }$ around the best-fitting shower. To increase accuracy of this fit, we simulate showers more densely in a region of $\pm 20 \mathrm{~g} / \mathrm{cm}^{2}$ around the $X_{\max }$ estimate, and redo the simulations if the new $X_{\max }$ estimate falls outside this range.

On average, the precision of the $X_{\max }$ estimate is $19 \mathrm{~g} / \mathrm{cm}^{2}$. Uncertainties in $X_{\max }$, primary energy, and shower core position are estimated from a Monte Carlo setup, where we reconstruct each simulated shower using the other showers in the ensemble. The precision in core reconstruction ranges from below 1 to about $3 \mathrm{~m}$ for strong showers coming in near the center of the array. We set a cutoff for the core precision of $7.5 \mathrm{~m}$ to reject showers with poor reconstructions. This value is a trade-off between including more showers, and having a higher precision in $X_{\max }$ and energy.

Systematic uncertainties amount to $7 \mathrm{~g} / \mathrm{cm}^{2}$ on $X_{\max }$, arising from the choice of hadronic interaction model in the simulations, remaining uncertainties in the atmospheric parameters, and a bound on the (residual) bias on the average $X_{\max }$ after sample selection. For the primary energy, the systematic uncertainty amounts to $14 \%$, mainly from the absolute calibration of the antennas [7].

We have tested for a possible general bias in the reconstruction process, using the simulated ensembles. For this we have used the dataset passing all criteria, taking all simulated showers within the densely simulated $X_{\max }$ range, to mimic the case of reconstructing real measurements.

The results are shown in Fig. 2 (left), where ' $X_{\max }$ bias' is defined as the reconstructed minus the real $X_{\max }$. On average, the bias amounts to $-2.9 \mathrm{~g} / \mathrm{cm}^{2}$, with a median of $-2.1 \mathrm{~g} / \mathrm{cm}^{2}$. This is of the same magnitude as the uncertainty on the overall $X_{\max }$ average, which is around $3.5 \mathrm{~g} / \mathrm{cm}^{2}$. Also, it is in line with the contribution of $3.3 \mathrm{~g} / \mathrm{cm}^{2}$ we added to the systematic uncertainties, accounting for possible bias on $\left\langle X_{\max }\right\rangle$. The running average deviates for the lowest region in $X_{\max }$, below about $570 \mathrm{~g} / \mathrm{cm}^{2}$. However, the average is based on a rather small number of showers in this region, hence it is as yet unclear if this signals an actual bias in this region. Should this bias be real, its effect on the current dataset is small. For a larger dataset it is worth to revisit this. 


\section{Sample selection}

For a mass composition analysis, we need to obtain a fiducial (unbiased) sample representing the natural $X_{\max }$ distribution. Bias arises from the triggering thresholds of the particle detectors and the radio antennas. The number of particles reaching the detectors is larger for showers with a maximum deep in the atmosphere, i.e. high $X_{\max }$ and/or lower zenith angle. As a result, for showers near the detection threshold there is a preference to detect high- $X_{\max }$ showers, and miss showers with lower $X_{\max }$. The detection threshold for the radio antennas is an opposite source of bias, as low- $X_{\max }$ showers spread their radio energy over a larger area, and are hence more easily detected in multiple LOFAR stations.

To obtain a minimally biased sample, we test for each measured shower whether a shower with the same parameters (energy, core position) would have been detected for any other natural $X_{\max }$ level, both in radio and in the particle detectors. The simulated ensemble provides the information needed for this test. The radio signal strength from CoREAS is compared to the LOFAR noise level in the measured shower, testing if it would trigger from an SNR criterion. The particle content from Corsika is fed into a detector simulation using Geant4 [11], yielding the expected energy deposit in each detector. From this, we test whether the particle array would trigger with high probability.

A setup like this is favored above a construction of a fiducial volume in parameter space, due to the irregularity of the antenna layout at LOFAR, and given the modest size of the dataset. We have opted for an 'on/off' approach where showers are either included or excluded; we do not attempt to keep showers in by accounting for diminishing detection efficiency beyond the edges of the unbiased volume. To further avoid dependence on uncertainties in the detector descriptions, the threshold levels are set slightly more conservative than the best estimate, and small variations in threshold levels do not significantly affect the results. Thus, uncertainties on e.g. the number of particles at the detectors, stemming from the unknown primary particle and from the hadronic interaction models, are also covered.

To see the effect of the fiducial selection, we show the following plots. From recalculating the average $X_{\max }$ versus energy with one or both bias tests disabled, it is seen in Fig. 2, right panel, that the effects of the fiducial selection are generally at or below the $10 \mathrm{~g} / \mathrm{cm}^{2}$ level. In Fig. 3 , we have plotted a quantity $Y_{\max }$, which is $X_{\max }$ corrected for generic energy dependence, i.e. the elongation rate which we take as $57 \mathrm{~g} / \mathrm{cm}^{2}$. This value is a reasonable approximation from Corsika simulations at constant composition; as the difference between average $X_{\max }$ and $Y_{\max }$ is relatively small, second-order effects from composition possibly varying with energy are negligible here.

Without the selection criteria, a deviation from the overall average is seen towards higher zenith angle (higher $1-\cos \theta$ ), left panel of Fig. 3. The fourth bin is then significantly away from the average, the fifth bin is inconclusive due to lack of data. This is mostly the result of disabling the particle bias test. In the selected set, the fourth bin contains only 22 showers and is about $1.5 \sigma$ above the baseline, which is inconclusive for residual bias. The highest two bins comprise $9 \%$ of the dataset, hence they have little influence on the average $X_{\max }$. In the right panel, the same is shown for the set of rejected showers. Here, we see a clear positive trend with zenith angle. This is expected, as for low zenith angles the bias is mainly downward (radio trigger), while at high zenith angles it is generally upward (particle trigger).

A simple set of cross-checks is to split the sample into two bins, evaluating $\left\langle X_{\max }\right\rangle$ for low 

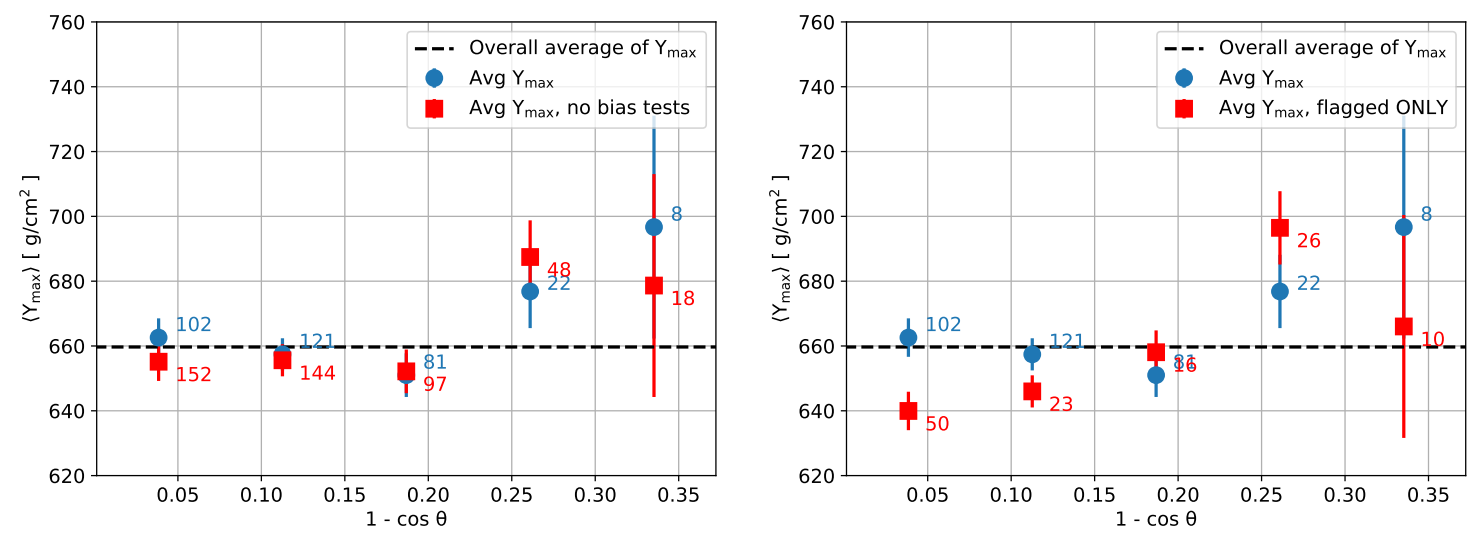

Figure 3: Left: the average $Y_{\max }$, which is an elongation-rate-corrected $X_{\max }$, as function of the cosine of the zenith angle $\theta$, with versus without fiducial selection criteria. Right: the same, showing only the rejected events in the red squares.

versus high values of a quantity such as the zenith angle. From random permutations of the data, one can assign a $p$ value to the observed difference. We have done this test for zenith angle, shower arrival date, air pressure at ground, and incoming celestial latitude. The resulting splits were within one-sigma, i.e. consistent with zero, for all but air pressure. For air pressure, the result was $p \approx 0.06$, hence also not significant.

\section{Results on $X_{\max }$ and mass composition analysis}

After the selection process, 334 showers remain in our dataset, out of 469 passing the criterion on core reconstruction precision. Their primary energy ranges from $10^{16.8}$ to $10^{18.3} \mathrm{eV}$. We have evaluated the average and standard deviation of $X_{\max }$, corresponding to the first two moments of the $X_{\max }$ distribution, in energy bins of width 0.25 in $\log _{10}(E / \mathrm{eV})$. They are plotted in Fig. 4, together with results from other cosmic-ray observatories.

The new result agrees reasonably well with the previous LOFAR analysis; the difference in the lowest-energy point is still statistically plausible, with $p=0.12$ for seeing at least such a difference (one-sided) in one out of three data points. Improved selection criteria and e.g. the improved energy estimate could also play a role here. Agreement is also found with other observatories for most energy bins; however, the difference with results from Pierre Auger Observatory is notable. Systematic uncertainties have been lowered to about $7 \mathrm{~g} / \mathrm{cm}^{2}$ in the present analysis, similar to the 8 to $10 \mathrm{~g} / \mathrm{cm}^{2}$ for Auger in this range.

Thus, there is tension in the results. It is at present unclear what causes the apparent difference. In principle, a true difference in mass composition between the northern and southern hemisphere is conceivable and could explain the findings. However, conclusions into this direction would at least require further detailed investigation into possible systematic differences, arising from the (considerably different) methods used to arrive at each result.

We have done a four-element mass composition analysis based on all information in the dataset, being energy and $X_{\max }$ per shower, and their respective uncertainties. This is achieved by a maximum 

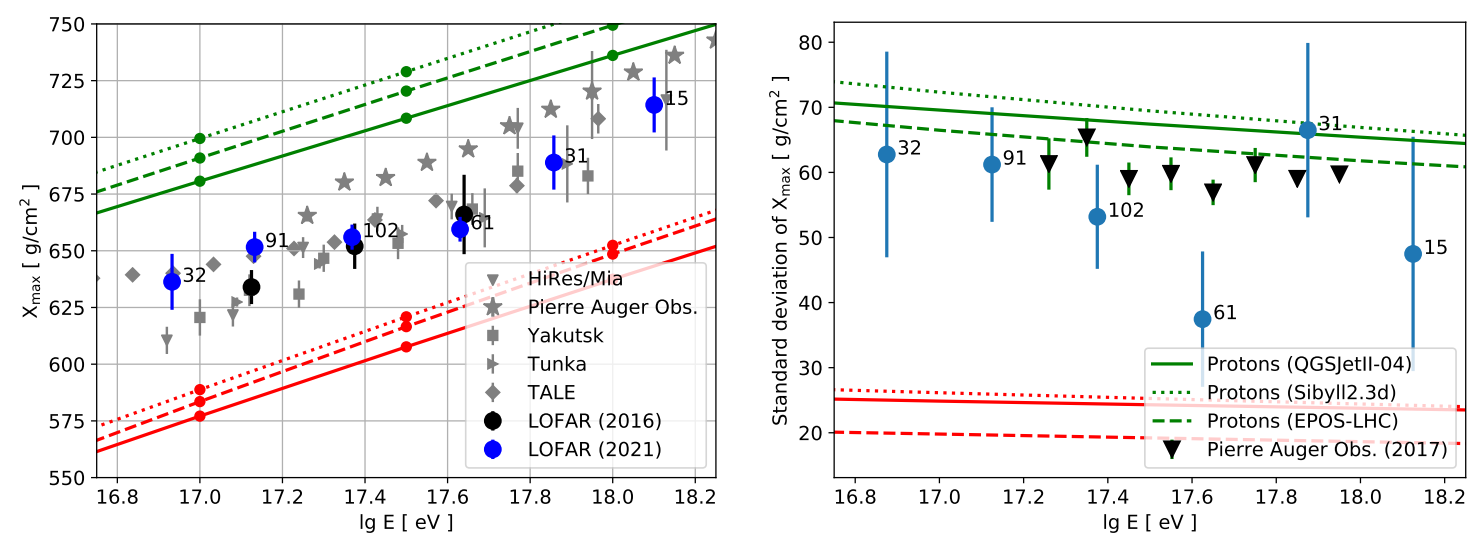

Figure 4: Left: Estimates for the mean $X_{\max }$ versus primary energy. For comparison, results are shown from Pierre Auger Observatory [12], HiRes/Mia [13], Yakutsk [14], Tunka [15], and TALE [16]. The colored lines refer to the average $X_{\max }$ for protons (green) and iron (red). Right: The same for the standard deviation of $X_{\max }$, compared to Pierre Auger Observatory.
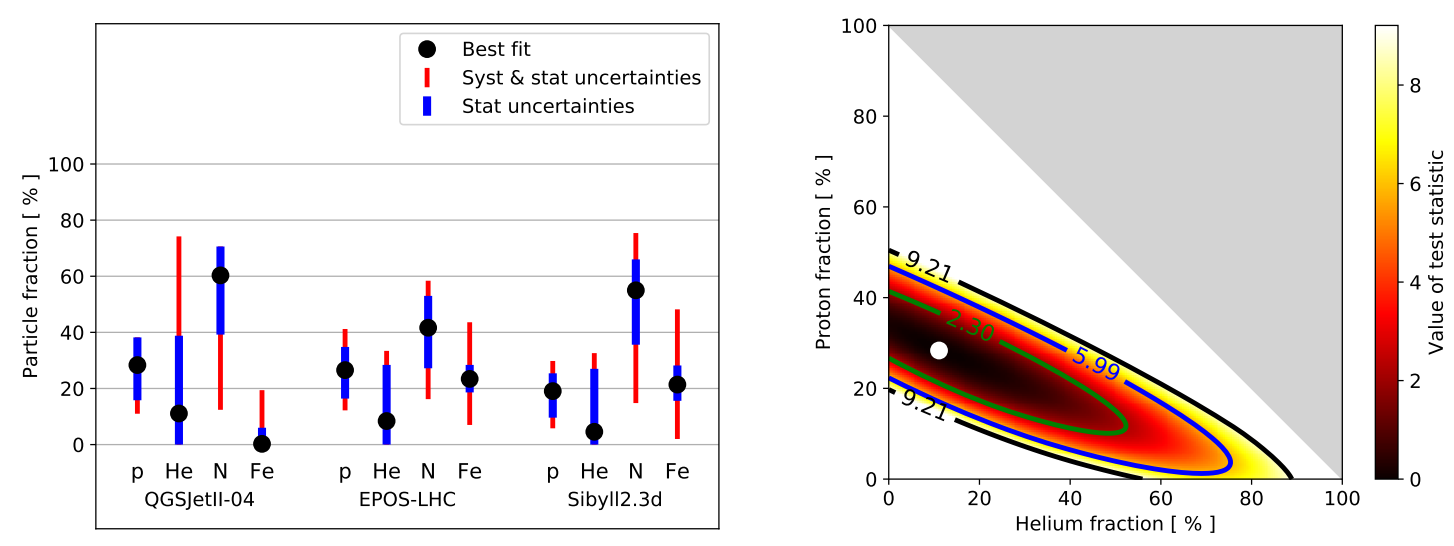

Figure 5: Left: Mass composition results for a four-element model, for three hadronic interaction models. Right: Contour plot for protons and helium, showing the allowed ranges within one-sigma, $95 \%$ and $99 \%$ confidence level, respectively.

likelihood analysis without binning in energy, using for each shower the $X_{\max }$ distributions at its respective energy level and uncertainties. The mass composition thus found applies to the entire dataset. This can be interpreted as a weighted average over the energy range, where the weight factors can be taken e.g. as the number of events per bin in Fig. 4. The 'center of mass' of the dataset is at $17.39 \pm 0.32$ in $\log _{10}(E / \mathrm{eV})$. Ideally, one would do the analysis separately in energy bins. However, due to the considerable overlap in the $X_{\max }$ distributions per element, an $N=334$ dataset is relatively modest, and further binning does not convey additional information [4].

We have used parametrizations of the $X_{\max }$ distributions per element, as from [17] and [18]. Results are shown in Fig. 5 (left), for three hadronic interaction models. A significant light-mass component is confirmed; would the proton fraction be considerably lower than the best fit, the helium fraction would be much higher, as shown in the contour plot, Fig. 5 (right). The intermediate-mass 
fraction, for which nitrogen is taken as a proxy in this model and comprising $\mathrm{C}, \mathrm{N}, \mathrm{O}$, and perhaps silicon, dominates.

When comparing results from the three hadronic interaction models, it is seen that within the given uncertainties, the differences are minor. At best fit, EPOS-LHC tends towards more iron (high-mass fraction), and Sibyll-2.3d tends towards more intermediate and higher-mass particles in general, compared to QGSJetII-04. Comparing the results to those from Pierre Auger Observatory, which are available in narrow energy bins, the results per element are in agreement within statistical and systematic uncertainties. Finding agreement in mass composition but not in average $X_{\max }$ is ascribed to additional uncertainties in a four-component model, as the level to which neighboring elements can be distinguished is limited (again due to the overlap in their natural $X_{\max }$ distribution).

\section{Summary}

We have presented an update of the analysis of the depth of shower maximum $X_{\max }$ and the mass composition of cosmic rays from LOFAR data, as well as a number of cross-checks not published earlier. Results for the average $X_{\max }$ as a function of energy are in agreement with the earlier LOFAR analysis, as well as most other observatories except the Pierre Auger Observatory. The difference with respect to the latter is outside the respective systematic uncertainty margins; the origin of this tension is not yet understood.

The mass composition as inferred from a four-element model shows a significant light-mass component, represented by protons and helium. At best fit, it amounts to about $40 \%$ assuming the QGSJetII-04 model for hadronic interactions, and about $25 \%$ for Sibyll-2.3d which generally yields the 'heaviest' composition. The fraction of intermediate-mass particles is dominant. Mass composition results are in agreement with those from Auger, unlike the average $X_{\max }$, as uncertainty margins are generally larger when aiming to distinguish elements in a four-component model.

From a variety of improvements to our analysis, systematic uncertainties have been lowered. These include accounting for the atmospheric conditions at each shower, improved fiducial selection criteria, and an absolute calibration of the antennas and signal chain. Systematic uncertainties amount to $7 \mathrm{~g} / \mathrm{cm}^{2}$ on $X_{\max }\left(9 \mathrm{~g} / \mathrm{cm}^{2}\right.$ in the mass composition analysis), and $14 \%$ on primary energy.

\section{Acknowledgements}

We acknowledge funding from the European Research Council under the European Union's Horizon 2020 research and innovation programme (grant agreement n. 640130). TNGT acknowledges funding from Vietnam National Foundation for Science and Technology Development (NAFOSTED) under grant number 103.01-2019.378. ST acknowledges funding from the Khalifa University Startup grant, project code 8474000237-FSU-2020-13.

LOFAR, the Low Frequency Array designed and constructed by ASTRON, has facilities in several countries, that are owned by various parties (each with their own funding sources), and that are collectively operated by the International LOFAR Telescope (ILT) foundation under a joint scientific policy. 


\section{References}

[1] M. P. van Haarlem et al., LOFAR: The Low Frequency Array, Astronomy and Astrophysics 556 (2013) A2.

[2] S. Buitink et al., Method for high precision reconstruction of air shower $X_{\max }$ using two-dimensional radio intensity profiles, Phys. Rev. D 90 (Oct, 2014) 082003.

[3] S. Buitink et al., A large light-mass component of cosmic rays at $10^{17}-10^{17.5}$ electronvolts from radio observations, Nature (Mar, 2016) 70 .

[4] A. Corstanje et al., Depth of shower maximum and mass composition of cosmic rays from 50 pev to 2 eev measured with the lofar radio telescope, Phys. Rev. D 103 (May, 2021) 102006.

[5] P. Mitra et al., Reconstructing air shower parameters with lofar using event specific gdas atmosphere, Astroparticle Physics 123 (2020) 102470.

[6] K. Mulrey et al., Calibration of the LOFAR low-band antennas using the Galaxy and a model of the signal chain, Astroparticle Physics 111 (2019) 1-11.

[7] K. Mulrey et al., On the cosmic-ray energy scale of the LOFAR radio telescope, Journal of Cosmology and Astroparticle Physics 2020 (nov, 2020) 017-017.

[8] D. Heck et al., CORSIKA: a Monte Carlo code to simulate extensive air showers. Feb., 1998.

[9] T. Huege, M. Ludwig, and C. W. James, Simulating radio emission from air showers with CoREAS, ARENA 2012, AIP Conf. Proc. 1535 (2013) 128-132.

[10] T. Bergmann et al., One-dimensional hybrid approach to extensive air shower simulation, Astroparticle Physics 26 (2007), no. 6420 - 432.

[11] S. Agostinelli et al., Geant4: a simulation toolkit, Nuclear Instruments and Methods in Physics Research Section A: Accelerators, Spectrometers, Detectors and Associated Equipment 506 (2003), no. 3250 - 303.

[12] A. Yushkov et al., Mass composition of cosmic rays with energies above $10^{17.2} \mathrm{eV}$ from the hybrid data of the Pierre Auger Observatory, Proceedings of the 36th International Cosmic Ray Conference, Madison, USA, PoS (2019) 482.

[13] P. Sokolsky, Final results from the High solution Fly's Eye (HiRes) Experiment, Nuclear Physics B - Proceedings Supplements 212-213 (2011) 74 - 78. Proceedings of the Cosmic Ray International Seminars (CRIS 2010).

[14] S. Knurenko et al., Mass composition of cosmic rays in the energy region $10^{16}-10^{18} \mathrm{eV}$, Proceedings of the 34th International Cosmic Ray Conference, The Hague, The Netherlands, PoS (2015) 254.

[15] V. Prosin et al., Primary CR energy spectrum and mass composition by the data of Tunka-133 array, EPJ Web of Conferences 99 (2015) 04002.

[16] Telescope Array Collaboration, The cosmic-ray composition between $2 \mathrm{PeV}$ and $2 \mathrm{EeV}$ observed with the TALE detector in monocular mode, 2020.

[17] M. De Domenico, M. Settimo, S. Riggi, and E. Bertin, Reinterpreting the development of extensive air showers initiated by nuclei and photons, Journal of Cosmology and Astroparticle Physics 2013 (2013), no. 07050.

[18] S. Petrera, Pers. communication, . 